\title{
Atitudes em Relaçáo às Pessoas com Deficiência: uma Revisão SisTEMÁTICA DA LITERATURA ${ }^{1,2}$ \\ Attitudes Towards People With Disabilities: a Systematic Literature REVIEW
}

\author{
Nuno LEAL ${ }^{3}$ \\ Celeste EUSÉBIO ${ }^{4}$ \\ Maria João da ROSA ${ }^{5}$
}

\begin{abstract}
RESUMO: A área de investigação do turismo acessível (TA) apresenta uma tendência de crescimento. Contudo, a maioria dos estudos foca-se na acessibilidade física, havendo poucos que analisem as atitudes dos estudantes e profissionais desse setor relativamente às pessoas com deficiência $(\mathrm{PcD})$. Assim sendo, esta revisão tem como objetivo analisar os trabalhos efetuados em outras áreas científicas sobre as atitudes relativamente às PcD. Pretende-se mapear metodologias, instrumentos de medição e principais variáveis e fatores associados às atitudes, para promover a sua inclusão na sociedade. Realizaram-se pesquisas na Scopus, tendo-se obtido 492 registos. Destes, foram selecionados 96 artigos para análise. Os resultados evidenciam que a avaliação das atitudes relativamente às $\mathrm{PcD}$ é uma temática de grande relevância em várias áreas científicas. Os estudos utilizam diversos instrumentos para medir essas atitudes. As experiências anteriores, qualidade e frequência do contato com PcD e conhecimento acerca da deficiência têm influência nas atitudes. Contudo, os resultados divergem na influência do perfil sociodemográfico nas atitudes face às PcD. Existe tendência para o recurso a metodologias quantitativas utilizando, como instrumento de recolha de dados, o questionário. O artigo termina identificando áreas de investigação relevantes para o aumento do conhecimento dos fatores que influenciam essas atitudes e, consequentemente, para o desenvolvimento do TA.
\end{abstract}

PALAVRAS-CHAVE: Turismo acessível. Escala de atitudes. Pessoas com deficiência. Determinantes das atitudes. Revisão bibliográfica.

ABSTRACT: The investigation area of accessible tourism (AT) has a growing trend. However, most studies focus on physical accessibility, and only a few analyse the attitudes of students and professionals in this sector towards people with disabilities $(\mathrm{PwD})$. This review aims to analyse the work carried out in other scientific areas on attitudes towards PwD. It is intended to map methodologies, measurement instruments and main variables and factors related with attitudes, to promote their inclusion in society. Research was carried out at Scopus and 492 records were obtained. Of these, 96 articles were selected for analysis. Results show that the assessment of attitudes towards $\mathrm{PwD}$ is a topic of great relevance in several scientific areas. Studies use several instruments to measure these attitudes. Previous experiences, quality, and frequency of contact with PwD and knowledge about disability have an influence on attitudes. However, results differ in the influence of the sociodemographic profile on attitudes towards PwD. There is a trend towards the use of quantitative methodologies, using the questionnaire as a tool for data collection. The article ends by identifying areas of research relevant to the increasing knowledge of the factors that influence these attitudes and, consequently, to the development of AT.

KEYWORDS: Accessible tourism. Attitudes scale. People with disabilities. Determinants of attitudes. Literature review.

\footnotetext{
${ }^{1}$ https://doi.org/10.1590/1980-54702020v26e0062

${ }^{2}$ Este trabalho foi desenvolvido no âmbito do projeto de investigação ACTION - POCI-01-0145-FEDER-030376 -, financiado pelo FEDER, por meio do COMPETE2020 - Programa Operacional Competitividade e Internacionalização (POCI), e por fundos nacionais (OE), por intermédio da FCT/MCTES.

${ }^{3}$ Departamento de Economia, Gestấo, Engenharia Industrial e Turismo. Universidade de Aveiro. Aveiro/Portugal. E-mail: cunha. leal@ua.pt. ORCID: https://orcid.org/0000-0002-2670-6285

${ }^{4}$ Departamento de Economia, Gestão, Engenharia Industrial e Turismo. Universidade de Aveiro. Unidade de Investigação em Governança, Competitividade e Políticas Públicas (GOVCOPP). Aveiro/Portugal. E-mail: celeste.eusebio@ua.pt. ORCID: https:// orcid.org/0000-0002-2220-5483

${ }^{5}$ Departamento de Economia, Gestão, Engenharia Industrial e Turismo. Universidade de Aveiro. Unidade de Investigação de Políticas do Ensino Superior (CIPES). Aveiro/Portugal. E-mail: m.joao@ua.pt. ORCID: https://orcid.org/0000-0003-2956-6801
} 


\section{INTRODUÇÁo}

O conceito de turismo acessível diz respeito ao processo colaborativo estabelecido entre os mais diversos atores do sistema turístico, com o objetivo de promover a adaptação da oferta turística a todos os turistas, de acordo com as suas necessidades de acesso, permanentes ou temporárias, visíveis ou invisíveis, mais ou menos severas, de forma a que possam dela usufruir em autonomia, igualdade e dignidade, sem barreiras físicas ou relacionadas aos serviços, produtos e ambientes (World Tourism Organization [UNWTO], 2013). Grande parte dos trabalhos de investigação científica, nessa área, centram o seu foco nas acessibilidades físicas. Um número limitado de estudos analisa as relaçóes interpessoais entre as pessoas com deficiência $(\mathrm{PcD})$ e os profissionais da indústria turística, mais propriamente as atitudes destes últimos em relação às $\mathrm{PcD}$ (Shaw $\&$ Coles, 2004). Sendo este um terreno em que a produção científica na área do turismo é escassa, faz todo o sentido investigar o que se faz em outras áreas científicas e setores de atividade, cujos serviços envolvem o contato permanente com PcD.

A deficiência é descrita como um fenómeno multidimensional que resulta da interação entre as pessoas e o ambiente físico e social. Assim, a incapacidade e a funcionalidade são influenciadas pela interação entre as condiçóes de saúde e os fatores contextuais, os quais podem ser humanos ou ambientais (Organizaçáo Mundial da Saúde [OMS], 2004). As atitudes em relação às $\mathrm{PcD}$ resultam da interação entre elas e outras pessoas, com ou sem deficiência, que se encontram no mesmo meio ambiente social. De acordo com Fazio e Olson (2003), essas atitudes advêm da ação isolada, ou por combinação de três componentes: (i) a componente afetiva (envolve a parte sentimental da pessoa); (ii) a componente cognitiva (envolve as crenças e os conhecimentos pessoais); e (iii) a componente comportamental (a forma como a atitude influencia a ação ou o comportamento).

As atitudes relativas a um determinado estímulo (objeto ou grupo social) assumem sempre um caráter avaliativo que pode ser positivo ou negativo (Allport, 1935), de maior ou menor intensidade (Fishbein \& Ajzen, 1975), servindo determinadas funçóes que auxiliam a resposta da pessoa, e a sua adaptação, ao ambiente social que a rodeia (Katz, 1960).

As atitudes negativas, em relação às $\mathrm{PcD}$, representam uma das maiores barreiras à sua participação em atividades sociais, como, por exemplo, o acesso aos serviços públicos de saúde, educação, lazer, recreio e desporto, contribuindo ainda mais para os efeitos incapacitantes da deficiência (Duckworth, 1988), reduzindo as oportunidades de integração social e promovendo o aprofundamento da sua condiçáo (Paris, 1993). Já a presença de atitudes positivas contribui, de forma inequívoca, para a integração e participação das PcD nas atividades sociais anteriormente referidas, melhora a qualidade dos serviços prestados e diminui o estigma das $\mathrm{PcD}$, sendo, atualmente, uma das principais preocupaçóes que a sociedade procura enfrentar e resolver (Fitzsimmons \& Barr, 1997).

Em 1928, Thurstone concluiu que "as atitudes podem ser medidas" (1928, p. 529), o que, inevitavelmente, "abriu portas a um dos mais importantes construtos da psicologia social" (Gawronski, 2007, p. 573). Desde então, foram inúmeras as tentativas de desenhar um modelo de medição das atitudes em relaçáo às $\mathrm{PcD}$, todos elas baseadas nas mais diversas teorias. Contudo, a grande maioria desses instrumentos apresentam alguns problemas relacionados à sua fiabilidade (consistência nas medições), flexibilidade de uso (poderem ser utilizados em dis- 
tintos momentos de recolha de dados) ou confiança na sua utilização (frequência de utilização em estudos) (Lam et al., 2010).

De acordo com Findler, Vilchinsky e Werner (2007), existem dois grupos de técnicas de medição: as diretas, autodescritivas ou explícitas, nas quais se pergunta diretamente à pessoa qual a sua atitude face a determinado objeto, e as indiretas ou implícitas, em que a atitude é aferida a partir de outro indicador que não a resposta (o que implica a ausência de controlo na resposta, ausência de intencionalidade, ausência de consciência e eficiência do processamento cognitivo que permite a sua ocorrência com baixos recursos cognitivos).

De forma a aumentar o conhecimento sobre as metodologias utilizadas para medir as atitudes face às $\mathrm{PcD}$, este estudo de revisão sistemática da literatura pretende efetuar uma recolha dos principais instrumentos, e das metodologias utilizadas em diversas áreas científicas, para medir as atitudes em relação às $\mathrm{PcD}$, bem como identificar as principais variáveis que as explicam. Por meio do mapeamento das descobertas já alcançadas pelas investigaçóes desenvolvidas, sobretudo em outras áreas do conhecimento, e em outras atividades econômicas, pretende-se, com esta revisão sistemática, identificar lacunas e oportunidades em termos de investigação científica sobre as barreiras atitudinais no âmbito do turismo acessível.

\section{Desenvolvimento}

Nesta seção, abordar-se-á a metodologia utilizada nesta pesquisa, que envolveu duas etapas descritas na sequência.

\subsection{Metodologia}

Esta revisão sistemática da literatura tem como objetivo analisar os estudos que vêm sendo publicados na literatura científica relacionados às atitudes de estudantes ou profissionais de vários setores em relação às $\mathrm{PcD}$, no sentido de identificar as áreas científicas em que eles têm sido realizados, as metodologias que têm sido utilizadas, bem como os fatores que influenciam as atitudes de estudantes e profissionais face às $\mathrm{PcD}$.

A metodologia utilizada integra duas etapas: (i) identificação dos estudos a analisar e (ii) descrição do processo a utilizar para analisar o conteúdo dos estudos identificados.

\subsubsection{Primeira eTAPa - IDENTIFICAÇáo dos ESTUdOS A ANALISAR}

Nesta revisão sistemática da literatura, privilegiou-se a base de dados Scopus, uma das maiores do mundo, que aloja artigos previamente revistos por outros investigadores (Elsevier, 2019), tendo-se utilizado, como protocolo de pesquisa, o processo descrito no Quadro 1. 


\begin{tabular}{|l|l|c|}
\hline Termos da pesquisa & $\begin{array}{l}\text { ( TITLE-ABS-KEY ( "attitudes" ) AND TITLE-ABS-KEY( "disabled people" ) } \\
\text { OR TITLE-ABS-KEY ( "people with disabilit*" ) OR TITLE-ABS-KEY ( "people } \\
\text { with special needs" ) AND TITLE-ABS-KEY ( "training" ) OR TITLE-ABS- } \\
\text { KEY ("education" ) }\end{array}$ \\
\hline Base de dados & Scopus \\
\hline Campos de pesquisa & Artigo e revisão de artigos \\
\hline Resultados & 492 \\
\hline Horizonte temporal & $1973-2019$ & Resultado \\
\hline Data da pesquisa & $09 / 05 / 2019$ & 492 \\
\hline \multicolumn{1}{|c|}{ Critério } & \multicolumn{1}{|c|}{ Filtro } & 492 \\
\hline Idioma & Inglês, francês, espanhol e português & 148 \\
\hline Tipo de documento & Artigos e revisões \\
\hline Temática analisada & Título e resumo & \multicolumn{2}{|c|}{} \\
\hline
\end{tabular}

Quadro 1. Resumo do protocolo de pesquisa, critérios e filtros de seleção dos documentos. Fonte: Elaborado pelos autores.

Conforme se pode observar no Quadro 1, a recolha dos dados foi efetuada em 9 de maio de 2019. Os resultados obtidos permitem observar que o primeiro documento identificado foi publicado em 1973. Por forma a captar todos os tipos de documentos relacionados com a temática em estudo, foram utilizadas, na pesquisa, as seguintes palavras: "attitudes", "disabled people", "people with disabilit*", "people with special needs" associadas a "education", "training" e "undergraduate*" e "graduate*". A investigação inclui todas as áreas, uma vez que, como exposto anteriormente, a produção científica com enfoque nesse tema é relativamente escassa.

Posteriormente, e tal como exposto no Quadro 1, definiram-se os critérios de inclusão ou exclusão dos documentos. Assim, como filtro, restringiu-se a pesquisa aos documentos cujo idioma era o inglês, francês, espanhol ou português. Seguidamente, efetuou-se uma análise de conteúdo com base no título e resumo de cada documento, eliminando-se todos aqueles que não correspondessem ao tema principal desta investigação.

Como exemplo de motivos de exclusão de artigos, há de destacarem-se aqueles que abordam temas que não se enquadram nos objetivos da presente revisão, tais como artigos cujo foco de análise se centra na medição dos impactos das políticas públicas de inclusão (Fisher $\&$ Purcal, 2017), de avaliação da satisfação e qualidade dos cuidados prestados, de identificação de barreiras ou dos seus impactos na comunidade. Foram, também, excluídos todos aqueles cujos objetos de estudo eram diferentes do estabelecido para esta revisão.

Conforme se pode observar na Figura 1, dos 148 artigos previamente selecionados, conseguiram-se obter 114 com texto completo, dos quais foram excluídos 18, por apresentarem temas que não se enquadravam com o objetivo da presente revisão: avaliação de políticas inclusivas, temas associados à religião ou com outra abordagem temática pouco relevante para o presente estudo. Assim, para esta revisão sistemática da literatura, foram selecionados 96 registos. 


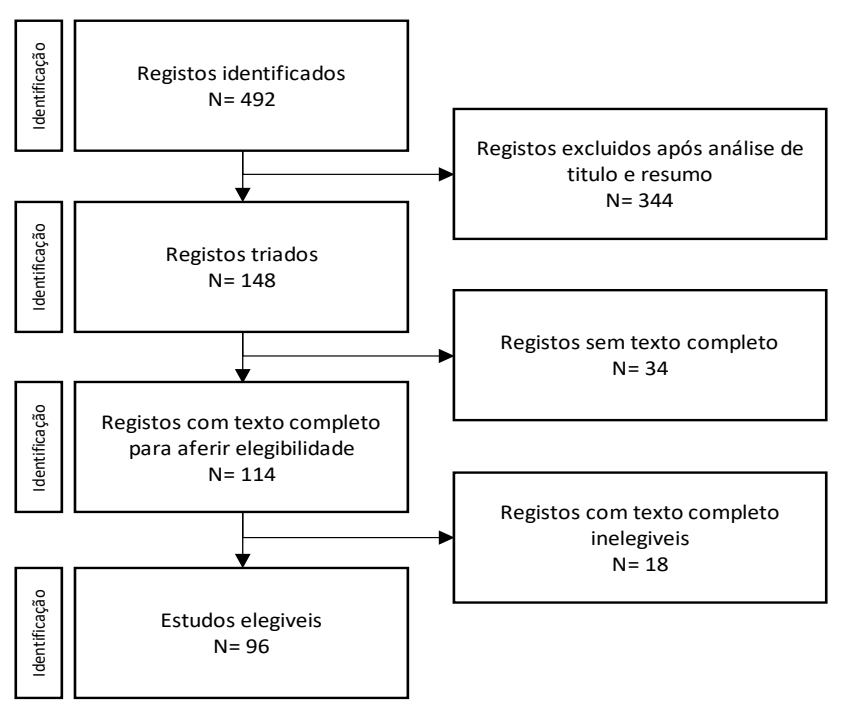

Figura 1. Etapas de seleção dos documentos Fonte: Elaborada pelos autores.

\subsubsection{SEGUNDA ETAPA - ANÁLISE DE CONTEÚDO}

Foi efetuada uma análise qualitativa do conteúdo dos 96 artigos científicos selecionados de acordo com o procedimento descrito na primeira etapa. A informação extraída de cada um foi catalogada em tabelas de resumo tendo em consideração os principais conceitos, variáveis, escalas e metodologias utilizadas em termos de recolha e análise de dados.

\section{Resultados E Discussáo}

Nesta seção, apresentam-se as áreas de estudo da produção científica analisada, as revistas científicas em que os artigos foram publicados, a distribuição geográfica das amostras, as temáticas dos artigos analisados, as tipologias de deficiência abordadas, a metodologia utilizada para recolha de dados, os instrumentos de medição de atitudes face às $\mathrm{PcD}$ e, por fim, os fatores que influenciam as atitudes.

\section{1 ÁREAS DE ESTUDO}

Como se pode observar no Tabela 1, as publicaçóes de artigos científicos relacionadas às atitudes de estudantes e profissionais em relação às $\mathrm{PcD}$ estão, na sua maioria, ligadas às áreas da Saúde e da Educação, concentrando a sua produçáo nas últimas duas décadas. 


\begin{tabular}{lcccccc}
\hline Área Científica & N estudos & $\mathbf{1 9 7 3 - 1 9 8 0}$ & $\mathbf{1 9 8 1 - 1 9 9 0}$ & $\mathbf{1 9 9 1 - 2 0 0 0}$ & $\mathbf{2 0 0 1 - 2 0 1 0}$ & $\mathbf{2 0 1 1 - 2 0 2 0}$ \\
\hline Saúde & 51 & 1 & 2 & 7 & 19 & 22 \\
Educação & 31 & - & 1 & 3 & 7 & 20 \\
Engenharia & 4 & - & - & - & 1 & 3 \\
Turismo & 2 & - & - & - & - & 2 \\
Economia e Gestão & 1 & - & - & - & - & 1 \\
Outras & 7 & - & - & - & 2 & 5 \\
\hline Total & $\mathbf{9 6}$ & $\mathbf{1}$ & $\mathbf{3}$ & $\mathbf{1 0}$ & $\mathbf{2 9}$ & $\mathbf{5 3}$ \\
\hline
\end{tabular}

Tabela 1. Investigações por área científica ao longo do tempo. Fonte: Elaborada pelos autores.

As áreas ligadas à saúde têm visto aumentar a sua produção científica acerca do tema em apreço, sobretudo nas últimas duas décadas. Por um lado, esse interesse pode estar associado ao novo paradigma que surgiu com a abordagem biopsicossocial da deficiência na Classificação Internacional de Funcionalidade, Incapacidade e Saúde (CIF), em detrimento do modelo médico da deficiência predominante nas sociedades ocidentais (Daruwalla \& Darcy, 2005). Por outro lado, parece indicar uma maior sensibilização para inclusão das PcD na sociedade por meio da identificação e da resposta às suas necessidades específicas (WORLD HEALT ORGAZIZATION [WHO], 2011).

Relativamente à Educação, o principal motivo associado a esse aumento considerável de produção científica parece residir na maior sensibilização para o tema da inclusão de $\mathrm{PcD}$ no sistema educativo, e na preocupação que se verifica acerca da preparação dos professores e aceitaçáo dos outros estudantes, como destacam Cortés e Campos (2016) ao referirem a preocupação do sistema educativo espanhol com essa matéria.

Também na área ligada à engenharia e à arquitetura, foram identificados três estudos, cujo principal objetivo ambiciona avaliar a predisposição dos seus estudantes, e profissionais, para a utilização dos princípios do Desenho Universal nos seus trabalhos.

Em relação à área do turismo, identificaram-se apenas dois artigos, os quais abordam o potencial da educação como agente de alteração das atitudes em relação às $\mathrm{PcD}$, o que, obviamente, indica uma clara lacuna nessa área.

\subsection{REVISTAS}

A Tabela 2 exibe as principais revistas científicas, bem como a quantidade de artigos publicados em cada uma delas acerca do tema em apreço. Note-se que estas revistas científicas parecem considerar que as investigaçóes das atitudes em relação às $\mathrm{PcD}$ e as ações de formação, ou alteraçôes aos programas curriculares, são um tema com elevado interesse académico. 


\begin{tabular}{lcc}
\hline \multicolumn{1}{c}{ Revista } & N estudos & \% por revista \\
\hline Medical Education & 5 & 5,2 \\
Nurse Education Today & 4 & 4,2 \\
$\begin{array}{l}\text { Disability and Health Journal; Disability and Rehabilitation; European } \\
\text { Journal of Dental Education; European Journal of Special Needs Education; } \\
\text { International Journal of Rehabilitation Research; }\end{array}$ & 3 & 3,1 \\
$\begin{array}{l}\text { Health and Social Care in the community; Journal of Applied Social } \\
\text { Psychology; Rehabilitation Psychology; Revista Complutense de Educación; } \\
\text { Medical Teacher; Rehabilitation Couselling Bulletin }\end{array}$ & 2 & 2,1 \\
Other & 60 & 62,5 \\
\hline Total & 96 & 100,0 \\
\hline
\end{tabular}

Tabela 2. Principais revistas de publicação.

Fonte: Elaborada pelos autores.

Conforme se depreende da Tabela 2, as revistas científicas identificadas estão, na sua maioria, ligadas à educação, mais especificamente à área da saúde, ou apresentam objetivos intimamente ligados à deficiência, à reabilitação ou à ação social.

\subsection{DisTRIBUIÇÁo GEOGRÁFICA}

A Tabela 3 resume a distribuição geográfica das amostras utilizadas nos estudos reportados relativas aos artigos em análise. Os países ocidentais mais desenvolvidos, como os Estados Unidos da América, Reino Unido, Austrália e Espanha, foram aqueles onde se realizaram mais estudos sobre a temática. Do total dos estudos analisados, $50 \%$ foram realizados nesses países.

\begin{tabular}{lcc}
\hline \multicolumn{1}{c}{ País } & N estudos por país & \% por país \\
\hline EUA & $\mathbf{1 9}$ & $\mathbf{1 9 , 8}$ \\
Reino Unido & $\mathbf{1 4}$ & $\mathbf{1 4 , 6}$ \\
Austrália & $\mathbf{1 0}$ & $\mathbf{1 0 , 4}$ \\
Espanha & $\mathbf{6}$ & $\mathbf{6 , 3}$ \\
Israel; Nova Zelândia & 5 & 5,2 \\
Turquia; Irlanda & 4 & 4,2 \\
Hong Kong; Canadá & 3 & 3,1 \\
Outros & 23 & 23,9 \\
\hline
\end{tabular}

Tabela 3. Distribuição geográfica das amostras.

Fonte: Elaborada pelos autores.

Esses resultados sugerem que existe uma consciencialização crescente da importância que essa matéria representa, no sentido de melhor conhecer a realidade acerca das atitudes dos estudantes e profissionais de cada área científica em relação às $\mathrm{PcD}$ nos países mais desenvol- 
vidos, bem como das metodologias mais eficazes tradicionalmente utilizadas, quer para a sua mediação, quer para a sua alteração.

\subsection{TEMÁTICAS ANALISADAS}

No que diz respeito à abordagem temática da investigação (Tabela 4), nesta revisão, constatou-se que os estudos podem ser categorizados em três grupos: (i) estudos que analisam apenas as atitudes em relação às $\mathrm{PcD}$; (ii) estudos que analisam questôes relacionadas à formação dos estudantes e profissionais; e (iii) estudos que analisam questóes relacionadas às atitudes e à formação. Do primeiro grupo, fazem parte estudos que analisam apenas as atitudes (de profissionais ou estudantes) relativamente às $\mathrm{PcD}$. Desse grupo, fazem parte 41 dos artigos analisados, produzidos essencialmente nos últimos 20 anos. Esses estudos estão relacionados, principalmente, ao desenvolvimento ou à avaliação de instrumentos para medir as atitudes face às PcD (Faulks et al., 2018; González, Martínez, Alonso, Avi, \& Río, 2016; Holler \& Werner, 2018; Lam et al., 2010; Smith \& McCulloch, 1978) e com a análise dos fatores que as poderão influenciar.

\begin{tabular}{lccccc|c|c}
\hline \multirow{2}{*}{ Abordagem temática } & \multicolumn{4}{c}{ Período } & \multicolumn{3}{c}{ Total } \\
\cline { 2 - 9 } & $\mathbf{1 9 7 3 - 1 9 8 0}$ & $\mathbf{1 9 8 1 - 1 9 9 0}$ & $\mathbf{1 9 9 1 - 2 0 0 0}$ & $\mathbf{2 0 0 1 - 2 0 1 0}$ & $\mathbf{2 0 1 1 - 2 0 2 0}$ & N & \% \\
\hline Atitudes & 1 & - & 3 & 12 & 25 & 41 & 42,7 \\
Formação & - & - & 6 & 13 & 19 & 38 & 39,6 \\
Atitudes e Formação & - & 3 & 1 & 4 & 9 & 17 & 17,7 \\
\hline Total & 1 & 3 & 10 & 29 & 53 & 96 & 100,0 \\
\hline
\end{tabular}

Tabela 4. Abordagem temática das investigações.

Fonte: Elaborada pelos autores.

Do segundo grupo de estudos, fazem parte 38 dos artigos analisados, datados entre 1991 e 2020, cujo foco principal é a formação. Esses estudos analisam principalmente os impactos de programas de intervenção, curriculares, ou outras ações de formação e sensibilização, sobre as atitudes dos estudantes e profissionais em relação às $\mathrm{PcD}$. Por fim, o terceiro grupo de estudos aborda, em simultâneo, questóes relacionadas às atitudes face à $\mathrm{PcD}$ e à formação de profissionais, ou futuros profissionais (estudantes), que contactam com pessoas com essas características (Tabela 4).

\subsection{TIPOLOGIAS DE DEFICIÊNCIA ABORDADAS}

$\mathrm{Na}$ Tabela 5, encontram-se resumidas as tipologias de deficiências focadas nas investigaçôes. Nos 96 estudos de investigação selecionados, a grande maioria (74,0\%) não especifica qual o tipo de deficiência que está a ser objeto de análise. Dos estudos que especificam claramente qual o tipo de deficiência que está a ser objeto de análise, os segmentos que têm merecido mais atenção incluem as PcD cognitiva/intelectual/de aprendizagem (15,6\% dos estudos) e as PcD motoras (13,5\%). 


\begin{tabular}{lcc}
\hline Tipologia de deficiência & N estudos & $\mathbf{\%}$ \\
\hline Não especificada & 71 & 74,0 \\
Cognitiva/Intelectual/Aprendizagem & 15 & 15,6 \\
Motora & 13 & 13,5 \\
Auditiva & 5 & 5,2 \\
Visual & 4 & 4,2 \\
Sensorial & 0 & 0,0 \\
\hline Total & $\mathbf{9 6}$ & $\mathbf{1 0 0 , 0}$ \\
\hline
\end{tabular}

Tabela 5. Tipologia de deficiência focada nos estudos.

Fonte: Elaborada pelos autores.

\subsection{Metodologia de RECOlHa de DAdos}

A Tabela 6 ilustra os métodos utilizados nos estudos que são objeto de análise neste artigo de revisão. Conforme se pode observar, a metodologia quantitativa, com recolha de dados por meio de questionários, é a que tem sido mais utilizada neste tipo de estudos $(68,8 \%$ dos que foram analisados).

\begin{tabular}{lcc}
\hline Método e abordagem & N es tudos & $\%$ \\
\hline Quantitativa & $\mathbf{6 8}$ & $\mathbf{7 0 , 8}$ \\
Questionário & 66 & 68,8 \\
Análise de dados secundários & 1 & 1,0 \\
$\quad$ Experimental & 1 & 1,0 \\
\hline Qualitativa & $\mathbf{1 1}$ & $\mathbf{1 1 , 4}$ \\
$\quad$ Entrevista & 5 & 5,2 \\
Métodos mistos qualitativos & 1 & 1,0 \\
$\quad$ Estudos de caso & 4 & 4,2 \\
$\quad$ Análise de narrativas & 1 & 1,0 \\
\hline Mista & $\mathbf{1 1}$ & $\mathbf{1 1 , 5}$ \\
\hline Revisão & $\mathbf{6}$ & $\mathbf{6 , 3}$ \\
\hline Total & $\mathbf{9 6}$ & $\mathbf{1 0 0 , 0}$ \\
\hline
\end{tabular}

Tabela 6. Métodos e abordagem dos estudos.

Fonte: Elaborada pelos autores.

As metodologias qualitativas foram utilizadas em apenas $11,4 \%$ dos trabalhos analisados, sendo a entrevista $(5,2 \%)$ e o estudo de casos $(4,2 \%)$ as técnicas de recolha de dados mais utilizadas. Por outro lado, 11,5\% dos estudos analisados utilizam métodos mistos, e apenas 6,3\%, são estudos teóricos, maioritariamente estudos de revisão da literatura.

\subsection{InSTRUMENTOS DE MEDiçÃo DE ATITUdes FACE À PCD}

De acordo com Fisher e Purcal (2017), as atitudes pessoais e o comportamento estão correlacionados, mas não são sempre idênticos: uma pessoa pode pensar e sentir de uma determinada forma, mas agir de outra forma, por vezes completamente oposta. Nos estudos analisados neste artigo de revisão, foram identificados vários instrumentos para medir as atitudes face à PcD (Tabela 7). 
Os principais instrumentos utilizados para medir as atitudes são, maioritariamente, escalas de concordância ou discordância, em relação a determinadas situaçôes ou opiniōes. A Tabela 7 resume os 30 instrumentos de medição de atitudes que foram identificados nos estudos analisados. Desses instrumentos, a escala Attitudes Toward Disabled Persons (ATDP) (nos seus 3 formulários - O, A e B), desenvolvida por Yuker na década de 1960, reúne a preferência de cerca de 34,8\% dos autores, como instrumento principal de medição das atitudes, enquanto que outros preferem desenvolver instrumentos adaptados à situação específica que pretendem investigar.

\begin{tabular}{|c|c|c|c|}
\hline Instrumento & $\mathbf{N}$ & Freq. & $\%$ \\
\hline Attitudes Toward Disabled Persons - ATDP & 1 & 24 & 34,8 \\
\hline Teacher Efficacy for Inclusive Practices Scale - TEIP & 1 & 5 & 7,3 \\
\hline Scale of Attitudes Toward disabled Persons (SADP); Multidimensional Attitude Scale (MAS) & 2 & 4 & 5,8 \\
\hline Sentiments, Attitudes and Concerns about Inclusive Education Revised Scale (SACIE-R) & 1 & 3 & 4,4 \\
\hline $\begin{array}{l}\text { Attitudes to Disability Scale (ADS); Interaction with Disabled people (IDP); Modified Issues } \\
\text { in Disabiliy Scale (MIDS); (Disability Attitudes in Health Care (DAHC) }\end{array}$ & 4 & 2 & 2,9 \\
\hline $\begin{array}{l}\text { Attitudes towards Inclusion of Students with Disabilities in Physical Education (AISDPE); } \\
\text { Actitudes hacia las Personas com Discapacidad (APD); Attitudes Toward Educational } \\
\text { Inclusion (ATEI); Attitudes Toward Teaching Individuals with Disabilities in Physical } \\
\text { education (ATIPDPE); Chedoke-McMaster Attitudes Towards Children with Handicaps } \\
\text { (CATCH); Escala de Atctitudes hacia las Personas com Discapacidad (EAPD); Exercise } \\
\text { Barriers Scale (EBS); Filtering Unconsciousness Matching of Implicit Emotions (FUMIE); } \\
\text { Mental Retardation Attitude Inventory-Revised (MRAI-R); Physical Educators' Attitudes } \\
\text { Toward Teaching the Handicapped (PEATTH); Perceived Physical Abilitty (PPA); Physical } \\
\text { Self Presentation Confidence (PSPC); Rosenbers' Self-esteem Scale (RSES); Attitude Towards } \\
\text { Inclusive Education Scale (ATIES); Contact with Disabled Persons (CDP); Community Living } \\
\text { Attitudes Scale Mental Retardation (CLAS-MR); Disability Social Relationship Scale (DSRS); } \\
\text { Implicit Association Test (IAT); Mindful Attention Awareness Scale (MAAS); Schwartz } \\
\text { Value Survey (SVS); Community Living Attitudes Scale- Intellectual Disability (CLAS-ID) }\end{array}$ & 21 & 1 & 1,5 \\
\hline Total & 30 & 69 & 100,0 \\
\hline
\end{tabular}

Tabela 7. Instrumentos de medição de atitudes identificados. Fonte: Elaborada pelos autores.

Ainda como instrumento complementar (secundário), ou de validação de resultados obtidos com o instrumento principal, alguns autores privilegiaram o Teacher Efficacy for Inclusive Practices Scale (TEIP) (específico para medir a eficácia das práticas inclusivas dos professores), ou de medição das atitudes em relação a tipologias de PcD específicas como, por exemplo, o Community Living Attitudes Scale Mental Retardation (CLAS-MR), que mede as atitudes em relação às pessoas com problemas cognitivos ou de desenvolvimento intelectual.

Acresce referir que, entre os artigos analisados, seis tinham como objetivo testar e validar escalas desenvolvidas especificamente para medir as atitudes de populaçóes distintas em relação às $\mathrm{PcD}$, ou de testar a adaptação de escalas já desenvolvidas e validadas a outros populaçôes residentes, em outros pontos geográficos. 


\subsection{FATORES QUE INFLUENCIAM AS ATITUDES}

$\mathrm{Na}$ literatura que analisa as atitudes face à $\mathrm{PcD}$, são identificados vários fatores que as poderão influenciar. Os fatores mais referidos estão relacionados ao conhecimento que os profissionais e os estudantes possuem das necessidades desse grupo da população, o contato/ interação que já tiveram com PcD, nomeadamente a sua experiência anterior, o seu perfil sociodemográfico e o fato de já terem frequentado ações de formação sobre o tema (Tabela 8).

\begin{tabular}{lrrrrrrr}
\hline \multirow{2}{*}{ Categorias de fatores/variáveis } & \multicolumn{2}{c}{ Existe associação } & \multicolumn{2}{c}{ Não existe associação } & \multirow{2}{*}{$\mathbf{N}$} & \multirow{2}{*}{$\%$} \\
\cline { 2 - 5 } & $\mathbf{N}$ & $\mathbf{\%}$ & $\mathbf{N}$ & $\mathbf{\%}$ & & \\
\hline Contato/interação & $\mathbf{5 0}$ & $\mathbf{9 0 , 9}$ & $\mathbf{5}$ & $\mathbf{9 , 1}$ & $\mathbf{5 5}$ & $\mathbf{1 0 0 , 0}$ \\
Experiência anterior & 9 & 90,0 & 1 & 10,0 & 10 & 18,2 \\
Contato (qualidade e frequência) & 41 & 91,1 & 4 & 8,9 & 45 & 81,8 \\
\hline Conhecimento & $\mathbf{4 5}$ & $\mathbf{1 0 0 , 0}$ & - & - & $\mathbf{4 5}$ & $\mathbf{1 0 0 , 0}$ \\
Conhecimento acerca da deficiência & 45 & 100,0 & - & - & 45 & 100,0 \\
\hline Perfil sociodemográfico & $\mathbf{2 9}$ & $\mathbf{6 9 , 0}$ & $\mathbf{1 3}$ & $\mathbf{3 1 , 0}$ & $\mathbf{4 2}$ & $\mathbf{1 0 0 , 0}$ \\
Idade & 8 & 66,7 & 4 & 33,3 & 12 & 28,6 \\
Género & 11 & 61,1 & 7 & 38,9 & 18 & 42,8 \\
Habilitações Académicas & 10 & 83,3 & 2 & 16,7 & 12 & 28,6 \\
\hline Ações de formação & $\mathbf{2 1}$ & $\mathbf{1 0 0 , 0}$ & - & - & $\mathbf{2 1}$ & $\mathbf{1 0 0 , 0}$ \\
Simulação/Recriação & 12 & 100,0 & - & - & 12 & 57,1 \\
Vídeo/Narrativas & 6 & 100,0 & - & - & 6 & 28,6 \\
Outras & 3 & 100,0 & - & - & 3 & 14,3 \\
\hline
\end{tabular}

Tabela 8. Categorias dos fatores e variáveis que influenciam as atitudes face à PcD. Fonte: Elaborada pelos autores.

A Tabela 8 detalha também os estudos que abordam essas associaçóes. Relativamente aos estudos que analisaram a associação entre as experiências anteriores com $\mathrm{PcD}$ e as atitudes face a elas, grande parte desses estudos $(90,0 \%)$ conclui que as experiências anteriores podem exercer uma influência positiva nas atitudes, como referem Wagner e Stewart (2001) e Seccombe (2007a, 2007b). Contudo, de acordo com Richard et al. (2005), essa associação não é estatisticamente significativa.

Dos estudos analisados, 41 sugerem que a qualidade e a frequência de contato com PcD possui uma associação com as atitudes em relação às elas. De acordo com Au e Man (2006), a frequência e a intensidade do contato promovem as atitudes positivas em relação às PcD. No entanto, outros consideram que essa associação não é estatisticamente significativa (Duckworth, 1988; Kurita \& Kusumi, 2010; Sánchez \& Puerta, 2018).

Em relação ao conhecimento acerca da deficiência, a totalidade dos estudos analisados (45) apontam no sentido de que a informação é essencial para promover atitudes positivas face às PcD, tal como Di Nardo, Kudlacek, Tafuri e Sklenarikova (2014) sugerem.

Sobre o perfil demográfico, constata-se que as variáveis mais estudadas pelos autores, no que se refere à influência que podem ter nas atitudes face às $\mathrm{PcD}$, dizem respeito ao género, à idade e às habilitaçóes académicas. Em se tratando do género, os autores que investigaram essa matéria sugerem que o fato de pertencer ao sexo feminino está diretamente associado a 
atitudes mais positivas em relação às $\mathrm{PcD}$. Foram, no entanto, identificados sete estudos cujos resultados não corroboram a conclusão anterior.

No que diz respeito à idade, é de salientar que 8 dos 12 estudos que abordam esse assunto sugerem que a idade tem uma associação com as atitudes; assim, à medida que a idade aumenta as atitudes em relação às PcD tornam-se mais positivas. Contudo, Parasuram (2006) alerta que tanto os jovens professores com menos anos de serviço como os mais idosos apresentam atitudes mais positivas do que o grupo de professores com idades intermédias. Entretanto, em quatro dos estudos analisados, a associação entre idade e atitudes não é confirmada.

De acordo com os resultados apurados, em 10 dos estudos analisados, observa-se que as pessoas que possuem habilitações literárias superiores possuem atitudes mais positivas. Existem, porém, dois estudos que afirmam que essa variável não influencia as atitudes em relação às $\mathrm{PcD}$.

Em relação às ações de formação, identificaram-se aquelas com diferentes tipos de abordagem, as quais, em comum, partilham o objetivo de sensibilizar os estudantes ou profissionais e, consequentemente, promover alteraçóes atitudinais. Entre elas, destacam-se as simulaçóes, sobretudo baseadas em sessóes com uma duração temporal variável e pré-determinada. Estas, quando bem desenhadas, podem promover o aumento do conhecimento acerca das necessidades das PcD, melhorar as atitudes dos formandos, bem como a aceitação de PcD (Lindsay \& Edwards, 2013). Todavia, French (1992) considera que o foco excessivo nos problemas, nas dificuldades e nos exercícios de simulação podem providenciar informação errada e enganosa e incutir atitudes negativas em relação às $\mathrm{PcD}$ em vez de positivas.

$\mathrm{Na}$ Tabela 8, pode-se observar que todas as intervençóes identificadas possuem uma associação positiva com as atitudes face às PcD. Relativamente aos recursos utilizados nas ações de formação, nesta revisão, identificaram-se a "simulação ou a recriação", "vídeos e narrativas" e "outras". As primeiras foram referidas como tendo uma associação positiva com as atitudes por 12 estudos, e envolvem situaçóes em que os participantes assumem o papel de uma pessoa com deficiência, enfrentando, eles próprios, os problemas a que estes últimos estão expostos diariamente. A segunda diz respeito a vídeos, filmes e narrativas dos problemas experienciados por $\mathrm{PcD}$, tendo sido analisados seis estudos que mostram que esses tipos de formaçóes são eficazes na alteração das atitudes em relação às $\mathrm{PcD}$. Por fim, existem outras açôes de formação (3), baseadas nos pressupostos das teorias da "Self-image" (Cohen, Roth, York, \& Neikrug, 2012) ou "Selfefficacy" (Forlin, Cedillo, Romero-Contreras, Fletcher, \& Rodriguez Hernández, 2010; Loreman, Sharma, \& Forlin, 2013; Oswald \& Swart, 2011; Van Boxtel, Napholz, \& Gnewikow, 1995; Velonaki et al., 2015), utilizadas sobretudo em estudos relacionados à educação inclusiva, os quais revelaram uma associação positiva em relação às atitudes de inclusão de PcD.

\section{Conclusāo}

O principal objetivo desta revisão sistemática da literatura era mapear as principais tendências nas abordagens metodológicas nas áreas científicas que mais têm vindo a estudar o tema, os fatores que podem estar associados a atitudes (positivas ou negativas) face às PcD e os instrumentos usados para a sua medição. A literatura científica, com foco nas atitudes dos estudantes do Ensino Superior e profissionais na área do turismo relativamente às PcD é parca, 
pelo que houve a necessidade de pesquisar informação relevante em outras áreas científicas, cujos setores de atividade, pela sua natureza, implicam o contato com PcD.

Ficou claro que o tema em apreço é relevante, de forma transversal, à maioria das áreas científicas que ambicionam promover atitudes positivas de estudantes e profissionais em relação às $\mathrm{PcD}$ e, consequentemente, melhorar a qualidade dos serviços prestados, bem como fomentar a inclusão social dessas pessoas. Este é um elemento importante, uma vez que a presença de atitudes positivas em relação às $\mathrm{PcD}$ é percecionada como um elemento crucial na integração social dessas pessoas, aumentando as oportunidades desse grupo da população, contribuindo para a sua valorização (Fitzsimmons \& Barr, 1997).

Esta revisão permitiu concluir que a grande maioria dos estudos acerca das atitudes em relação às $\mathrm{PcD}$ são das áreas científicas ligadas à saúde, sobretudo nas áreas da enfermagem e medicina, e ainda da educação. Os estudos da área da saúde apontam como objetivo a melhoria da qualidade do serviço prestado e a promoção da integração das PcD na sociedade. Na área da educação, pretende-se, sobretudo, promover a inclusão de alunos com deficiência. Os estudos têm especial incidência temporal nas últimas duas décadas.

Relativamente à distribuição geográfica das amostras incluídas nos estudos analisados, verifica-se que elas se concentram sobretudo nos países mais desenvolvidos, encabeçado pelos EUA, Reino Unido, Austrália e Espanha, mas têm também expressão a nível global, o que pode indiciar que este campo de investigação se está a tornar uma nova tendência de interesse para os académicos.

No que diz respeito ao método de abordagem, ficou claro que a grande maioria faz uso de métodos quantitativos, utilizando tendencialmente um ou mais questionários para a recolha de dados. Por outro lado, a abordagem temática das investigaçóes cobre tanto a avaliação das atitudes em relação às $\mathrm{PcD}$, como a formação de estudantes e profissionais para lidarem com as PcD, ou mesmo em ambos os temas.

Grande parte dos artigos científicos analisados concluem que o conhecimento acerca do tema, bem como a qualidade e a frequência de contato com PcD, influenciam as atitudes em relação a estas últimas, no sentido de que, quanto maior for a exposição a estes fatores, mais positivas são as atitudes.

Tal como os autores Findler, Vilchinsky e Werner (2007) haviam sugerido, esta revisão evidenciou que existem dois grupos de técnicas de medição das atitudes face à PcD: as diretas, autodescritivas ou explícitas e as indiretas ou implícitas. Entretanto, esta revisão permitiu identificar três dezenas de instrumentos de medição de atitudes, sendo que a grande maioria dos estudos evidenciam preferência pela utilização da ATDP. Contudo, a maior parte dos estudos não especifica uma tipologia de deficiência, pelo que se assume que estas são avaliadas de forma geral. Esta revisão serve ainda de alerta para o setor do turismo ao trazer ao debate a importância do estudo das atitudes em relação às PcD, por parte dos estudantes do Ensino Superior (futuros profissionais do setor), que tanto tem sido negligenciado.

Esta revisão sistemática da literatura enfrentou algumas limitações que podem interferir nas conclusóes. Desde logo, a começar pela incapacidade de obter todos os artigos identificados na fase da primeira seleção, os quais poderiam acrescentar dados pertinentes. Todavia, 
as diferenças existentes entre as populaçóes em estudo podem enviesar os resultados quando da aplicação a estudos similares no setor do turismo, uma vez que, apesar do contato entre profissionais de cada ramo e as PcD ser um ponto em comum dos seus serviços, a natureza deles pode variar em razão da necessidade individual de cada pessoa.

\section{REFERÊNCIAS}

Allport, G. W. (1935). Attitudes. In S. T. Fiske, D. T. Gilbert, \& G. Lindzey (Eds.), Handbook of Social Psychology (v. 1, pp. 798-844). Clark University Press.

Au, K. W., \& Man, D. W. K. (2006). Attitudes toward people with disabilities: a comparison between health care professionals and students. International Journal of Rehabilitation Research, 29(2), 155160 .

Cohen, R., Roth, D., York, A., \& Neikrug, S. (2012). Youth leadership program for changing self-image and attitude toward people with disabilities. Journal of Social Work in Disability and Rehabilitation, 11(3), 197-218.

Cortés, E. G., \& Campos, S. R. (2016). ¿Barreras invisibles? Actitudes de los estudiantes universitarios ante sus compañeros con discapacidad. Revista Complutense de Educación, 27(1), 219-235.

Daruwalla, P., \& Darcy, S. (2005). Personal and societal attitudes to disability. Annals of Tourism Research, 32(3), 549-570.

Di Nardo, M., Kudlacek, M., Tafuri, D., \& Sklenarikova, J. (2014). Attitudes of preservice physical educators toward individuals with disabilities at University Parthenope of Napoli. Acta Gymnica, 44(4), 211-221.

Duckworth, S. C. (1988). The effect of medical education on the attitudes of medical students towards disabled people. Medical Education, 22(6), 501-505.

Elsevier. (2019). The largest database of peer-reviewed literature. Scopus: Elsevier Solutions.

Faulks, D., Dougall, A., Ting, G., Ari, T., Nunn, J., Friedman, C. ... Newton, J. T. (2018). Development of a battery of tests to measure attitudes and intended behaviours of dental students towards people with disability or those in marginalised groups. European Journal of Dental Education, 22(2), e278-e290.

Fazio, R. H., \& Olson, M. A. (2003). Attitudes: foundations, functions, and consequences. In M. A. Hogg, \& J. Cooper (Eds.), The sage handbook of Social Psychology (2nd ed., pp. 139-160). London: Sage Publications.

Findler, L., Vilchinsky, N., \& Werner, S. (2007). The multidimensional attitudes scale toward persons with disabilities (MAS). Rehabilitation Counseling Bulletin, 50(3), 166-176.

Fishbein, M., \& Ajzen, I. (1975). Attitude Formation. In M. Fishbein, \& I. Ajzen (Eds.), Belief, attitude, intention and behavior: an introdution to theory and research (pp. 216-287). Reading, MA: AddisonWesley Publishing Company, Inc.

Fisher, K. R., \& Purcal, C. (2017). Policies to change attitudes to people with disabilities. Scandinavian Journal of Disability Research, 19(2), 161-174.

Fitzsimmons, J., \& Barr, O. (1997). A review of the reported attitudes of health and social care professionals towards people with learning disabilities: Implications for education and further research. Journal of Learning Disabilities, 1(2), 57-64. 
Forlin, C., Cedillo, I. G., Romero-Contreras, S., Fletcher, T., \& Rodriguez Hernández, H. J. (2010). Inclusion in Mexico: Ensuring supportive attitudes by newly graduated teachers. International Journal of Inclusive Education, 14(7), 723-739.

French, S. (1992). Simulation exercises in disability awareness training: a critique. Disability, Handicap \& Society, 7(3), 257-266.

Gawronski, B. (2007). Editorial: attitudes can be measured! But what is an attitude?. Social Cognition, 25(5), 573-581.

González, V. A., Martínez, B. A., Alonso, M. A. V., Avi, M. R., \& Rio, C. J. (2016). Evaluación de actitudes de los profesionales hacia las personas con discapacidad. Siglo Cero. Revista Española Sobre Discapacidad Intelectual, 47(2), 7-41.

Holler, R., \& Werner, S. (2018). Perceptions towards disability among social work students in Israel: Development and validation of a new scale. Health and Social Care in the Community, 26(3), 423432.

Katz, D. (1960). The Functional Approach to the Study of Attitudes. Public Opinion Quarterly, 24(2), 163. DOI: https://doi.org/10.1086/266945

Kurita, T., \& Kusumi, T. (2010). Implicit and explicit attitudes toward people with disabilities and effects of the internal and external sources of motivation to moderating prejudice. Psychologia, 52(4), 253-260.

Lam, W. Y., Gunukula, S. K., McGuigan, D., Isaiah, N., Symons, A. B., \& Akl, E. A. (2010). Validated instruments used to measure attitudes of healthcare students and professionals towards patients with physical disability: A systematic review. Journal of NeuroEngineering and Rehabilitation, 7(1), 1-7.

Lindsay, S., \& Edwards, A. (2013). A systematic review of disability awareness interventions for children and youth. Disability and Rehabilitation, 35(8), 623-646.

Loreman, T., Sharma, U., \& Forlin, C. (2013). Do pre-service teachers feel ready to teach in inclusive classrooms? A Four Country Study of Teaching Self-efficacy. Australian Journal of Teacher Education, $38(1), 27-44$.

Organização Mundial da Saúde (2004). Classificação Internacional de Funcionalidade, Incapacidade e Saúde. Lisboa: DGS.

Oswald, M., \& Swart, E. (2011). Addressing South African pre-service teachers' sentiments, attitudes and concerns regarding Inclusive Education. International Journal of Disability, Development and Education, 58(4), 389-403.

Parasuram, K. (2006). Variables that affect teachers' attitudes towards disability and inclusive education in Mumbai, India. Disability and Society, 21(3), 231-242.

Paris, M. (1993). Attitudes of medical students and health care professionals toward people with disabilities. Archives of Physical Medicine and Rehabilitation, 74(8), 818-825.

Richard, I., Compain, V., Mouillie, J. M., Adès, F., Garnier, F., Dubas, F., \& Saint-André, J. P. (2005). Évaluation de l'attitude vis-à-vis des personnes handicapées des étudiants en médecine de 3e et 4e année par le questionnaire «attitude towards disabled persons». Effets de l'enseignement théorique et de stages dans les services de médecine physique et ré. Annales de Readaptation et de Medecine Physique, 48(9), 662-667. 
Sánchez, M. T. P., \& Puerta, M. A. (2018). Primeros pasos hacia la inclusión: actitudes hacia la discapacidad de docentes en educación infantil. Revista de Investigación Educativa, 36(2), 365-379.

Seccombe, J. A. (2007a). Attitudes towards disability in an undergraduate nursing curriculum: A literature review. Nurse Education Today, 27(5), 459-465.

Seccombe, J. A. (2007b). Attitudes towards disability in an undergraduate nursing curriculum: The effects of a curriculum change. Nurse Education Today, 27(5), 445-451.

Shaw, G., \& Coles, T. (2004). Disability, holiday making and the tourism industry in the UK: A preliminary survey. Tourism Management, 25(3), 397-403.

Smith, N. J., \& McCulloch, J. W. (1978). Measuring attitudes towards the physically disabled: Testing the "Attitude Towards Disabled Persons" scale (A.T.D.P. Form O) on social work and non-social work students. International Journal of Rehabilitation Research, 1(2), 187-197.

Thurstone, L. L. (1928). Attitudes Can Be Measured. American Journal of Sociology 33, 529-554.

Van Boxtel, A. M., Napholz, L., \& Gnewikow, D. (1995). Using a wheelchair activity as a learning experience for student nurses. Rehabilitation Nursing, 20(5), 265-272.

Velonaki, V. S., Kampouroglou, G., Velonaki, M., Dimakopoulou, K., Sourtzi, P., \& Kalokerinou, A. (2015). Nurses' knowledge, attitudes and behavior toward deaf patients. Disability and Health Journal, 8(1), 109-117.

Wagner, A. K., \& Stewart, P. J. B. (2001). An internship for college students in Physical Medicine and Rehabilitation - effects on awareness, career choice, and disability perceptions. American Journal of Physical Medicine \& Rehabilitation, 80, 459-465.

World Health Organization (2011). World report on disability 2011. American Journal of Physical Medicine Rehabilitation Association of Academic Physiatrists, 91, 549.

World Tourism Organization (2013). Recommendations on Accessible Tourism. Recuperado em 30 de março de 2020 de https://www.accessibletourism.org/resources/accesibilityen_2013_unwto.pdf

\section{REFERÊNCIAS DA AMOSTRA DA PESQUISA}

Abellán, J., Sáez-Gallego, N., \& Reina, R. (2018). Explorando el efecto del contacto y el deporte inclusivo en Educación Física en las actitudes hacia la discapacidad intelectual en estudiantes de secundaria. RICYDE. Revista Internacional de Ciencias Del Deporte, 14(53), 233-242.

Anderson, E. S., Smith, R., \& Thorpe, L. N. (2010). Learning from lives together: Medical and social work students' experiences of learning from people with disabilities in the community. Health and Social Care in the Community, 18(3), 229-240.

Au, K. W., \& Man, D. W. K. (2006). Attitudes toward people with disabilities: a comparison between health care professionals and students. International Journal of Rehabilitation Research, 29(2), 155160.

Aulagnier, M., Verger, P., Ravaud, J. F., Souville, M., Lussault, P. Y., Garnier, J. P., \& Paraponaris, A. (2005). General practitioners' attitudes towards patients with disabilities: The need for training and support. Disability and Rehabilitation, 27(22), 1343-1352.

Ay, P., Save, D., \& Fidanoglu, O. (2006). Does stigma concerning mental disorders differ through medical education? A survey among medical students in Istanbul. Social Psychiatry and Psychiatric Epidemiology, 41(1), 63-67. 
Bean, K. F., \& Hedgpeth, J. (2014). The Effect of Social Work Education and Self-Esteem on Students' Social Discrimination of People with Disabilities. Social Work Education, 33(1), 49-60.

Bizjak, B., Knežević, M., \& Cvetrežnik, S. (2011). Attitude change towards guests with disabilities. Reflections from Tourism Students. Annals of Tourism Research, 38(3), 842-857.

Brownlee, J., \& Carrington, S. (2000). Opportunities for authentic experience and reflection: A teaching programme designed to change attitudes towards disability for pre-service teachers. Support for Learning, 15(3), 99-105.

Bu, P., Veloski, J. J., \& Ankam, N. S. (2016). Effects of a brief curricular intervention on medical students' attitudes toward people with disabilities in healthcare settings. American Journal of Physical Medicine and Rehabilitation, 95(12), 939-945.

Byron, M., Cockshott, Z., Brownett, H., \& Ramkalawan, T. (2005). What does "disability" mean for medical students? An exploration of the words medical students associate with the term "disability". Medical Education, 39(2), 176-183.

Campbell, J., Gilmore, L., \& Cuskelly, M. (2003). Changing student teachers' attitudes towards disability and inclusion. Journal of Intellectual and Developmental Disability, 28(4), 369-379.

Carvalho-Freitas, M. N. de, \& Stathi, S. (2017). Reducing workplace bias toward people with disabilities with the use of imagined contact. Journal of Applied Social Psychology, 47(5), 256-266.

Castillo, J. L. Á., \& Fernández, M. B. (2015). Predictors of attitudes toward inclusion of students with special educational needs in future education professionals. Revista Complutense de Educacion, 26(3), 627-645.

Chrysostomou, M., \& Symeonidou, S. (2017). Education for disability equality through disabled people's life stories and narratives: working and learning together in a school-based professional development programme for inclusion. European Journal of Special Needs Education, 32(4), 572585 .

Cohen, R., Roth, D., York, A., \& Neikrug, S. (2012). Youth leadership program for changing self-image and attitude toward people with disabilities. Journal of Social Work in Disability and Rehabilitation, 11(3), 197-218.

Cortés, E. G., \& Campos, S. R. (2016). ¿Barreras invisibles? Actitudes de los estudiantes universitarios ante sus compañeros con discapacidad. Revista Complutense de Educación, 27(1), 219-235.

Di Nardo, M., Kudlacek, M., Tafuri, D., \& Sklenarikova, J. (2014). Attitudes of preservice physical educators toward individuals with disabilities at University Parthenope of Napoli. Acta Gymnica, 44(4), 211-221.

Dougall, A., Pani, S. C., Thompson, S., Faulks, D., Romer, M., \& Nunn, J. (2012). Developing an undergraduate curriculum in Special Care Dentistry - by consensus. European Journal of Dental Education, 17(1), 46-56.

Downs, P., \& Wiliams, T. (1994). Students attitudes toward integration of people with disabilities activity settings. Adapted Physical Activity Quartely, 11, 32-43.

Duckworth, S. C. (1988). The effect of medical education on the attitudes of medical students towards disabled people. Medical Education, 22(6), 501-505.

Dunn, D. S., Fisher, D. J., \& Beard, B. M. (2012). Revisiting the mine/thine problem: A sensitizing exercise for clinic, classroom, and attributional research. Rehabilitation Psychology, 57(2), 113-123. 
Edwards, D. M., Merry, A. J., \& Pealing, R. (2002). Disability Part 3: Improving access to dental practices in Merseyside. British Dental Journal, 193(6), 317-319.

Evcil, N. (2010). Designers' attitudes towards disabled people and the compliance of public open places: The case of Istanbul. European Planning Studies, 18(11), 1863-1880.

Faulks, D., Dougall, A., Ting, G., Ari, T., Nunn, J., Friedman, C. ... Newton, J. T. (2018). Development of a battery of tests to measure attitudes and intended behaviours of dental students towards people with disability or those in marginalised groups. European Journal of Dental Education, 22(2), e278-e290.

Fisher, K. R., \& Purcal, C. (2017). Policies to change attitudes to people with disabilities. Scandinavian Journal of Disability Research, 19(2), 161-174.

Fitzsimmons, J., \& Barr, O. (1997). A review of the reported attitudes of health and social care professionals towards people with learning disabilities: Implications for education and further research. Journal of Learning Disabilities, 1(2), 57-64.

Florian, V., \& Kehat, D. (1987). Changing High School Students' Attitudes Toward Disabled People. Health and Social Work, 12(1), 57-63.

Forlin, C., Cedillo, I. G., Romero-Contreras, S., Fletcher, T., \& Rodriguez Hernández, H. J. (2010). Inclusion in Mexico: Ensuring supportive attitudes by newly graduated teachers. International Journal of Inclusive Education, 14(7), 723-739

Freeman, A. (1988). Students' attitudes to disability. Journal of the British Institute of Mental Handicap (APEX), 16(3), 104-108.

French, S. (1992). Simulation Exercises in Disability Awareness Training: A critique. Disability, Handicap \& Society, 7(3), 257-266.

French, S. (1994). Attitudes of Health Professionals towards Disabled People A Discussion and Review of the Literature. Physiotherapy (United Kingdom), 80(10), 687-693.

Galli, G., Lenggenhager, B., Scivoletto, G., Molinari, M., \& Pazzaglia, M. (2015). Don't look at my wheelchair! The plasticity of longlasting prejudice. Medical Education, 49(12), 1239-1247.

Girli, A., Sarı, H. Y., Kırkım, G., \& Narin, S. (2016). University students' attitudes towards disability and their views on discrimination. International Journal of Developmental Disabilities, 62(2), 98107.

González, V. A., Martínez, B. A., Alonso, M. A. V., Avi, M. R., \& Rio, C. J. (2016). Evaluación de actitudes de los profesionales hacia las personas con discapacidad. Siglo Cero. Revista Española Sobre Discapacidad Intelectual, 47(2), 7-41.

Hassanein, E. E. A. (2015). Changing teachers' negative attitudes toward persons with intellectual disabilities. Behavior Modification, 39(3), 367-389.

Hayashi, R., \& Kimura, M. (2003). An Exploratory Study on Attitudes Toward Persons with Disabilities Among U.S. and Japanese Social Work Students. Journal of Social Work in Disability \& Rehabilitation, 2(2-3), 65-85.

Hein, S., Grumm, M., \& Fingerle, M. (2011). Is contact with people with disabilities a guarantee for positive implicit and explicit attitudes? European Journal of Special Needs Education, 26(4), 509522. 
Hitch, D., Dell, K., \& Larkin, H. (2016). Does universal design education impact on the attitudes of architecture students towards people with disability? Journal of Accessibility and Design for All, 6(1), $26-48$.

Holler, R., \& Werner, S. (2018). Perceptions towards disability among social work students in Israel: Development and validation of a new scale. Health and Social Care in the Community, 26(3), 423432 .

Hsu, T. H., Huang, Y. T., Liu, Y. H., Ososkie, J., Fried, J., \& Bezyak, J. (2015). Taiwanese attitudes and affective reactions toward individuals and coworkers who have intellectual disabilities. American Journal on Intellectual and Developmental Disabilities, 120(2), 110-124.

Ison, N., McIntyre, S., Rothery, S., Smithers-Sheedy, H., Goldsmith, S., Parsonage, S., \& Foy, L. (2010). "Just like you": A disability awareness programme for children that enhanced knowledge, attitudes and acceptance: Pilot study findings. Developmental Neurorehabilitation, 13(5), 360-368.

Keller, C., \& Siegrist, M. (2010). Psychological Resources and Attitudes Toward People. Journal of Applied Social Psychology, 40(2), 389-401.

Kritsotakis, G., Galanis, P., Papastefanakis, E., Meidani, F., Philalithis, A. E., Kalokairinou, A., \& Sourtzi, P. (2017). Attitudes towards people with physical or intellectual disabilities among nursing, social work and medical students. Journal of Clinical Nursing, 26(23-24), 4951-4963.

Kurita, T., \& Kusumi, T. (2010). Implicit and explicit attitudes toward people with disabilities and effects of the internal and external sources of motivation to moderating prejudice. Psychologia, 52(4), 253-260.

Kwon, K. A., Hong, S. Y., \& Jeon, H. J. (2017). Classroom readiness for successful inclusion: teacher factors and preschool children's experience with and attitudes toward peers with disabilities. Journal of Research in Childhood Education, 31(3), 360-378.

Lindsay, S., \& Edwards, A. (2013). A systematic review of disability awareness interventions for children and youth. Disability and Rehabilitation, 35(8), 623-646.

Loreman, T., Sharma, U., \& Forlin, C. (2013). Do pre-service teachers feel ready to teach in inclusive classrooms? A Four Country Study of Teaching Self-efficacy. Australian Journal of Teacher Education, 38(1), 27-44.

Lu, J., Webber, W. B., Romero, D., \& Chirino, C. (2017). Changing attitudes toward people with disabilities using public media: an experimental study. Rehabilitation Counseling Bulletin, 61(3), 175-186.

Lynch, J., Last, J., Dodd, P., Stancila, D., \& Linehan, C. (2018). 'Understanding Disability': Evaluating a contact-based approach to enhancing attitudes and disability literacy of medical students. Disability and Health Journal, 12(1), 65-71.

Lyon, L., \& Houser, R. (2016). Psychometric evaluation of the attitudes to disability scale for use with nurse educators. Journal of Nursing Measurement, 24(3), 465-476.

Mac Giolla Phadraig, C., Nunn, J. H., Tornsey, O., \& Timms, M. (2015). Does special care dentistry undergraduate teaching improve dental student attitudes towards people with disabilities? European Journal of Dental Education, 19(2), 107-112.

Mamboleo, G. I., Diallo, A., Ocharo, R. M., Oire, S. N., \& Kampfe, C. M. (2015). Socio-ecological influences of attitudes toward disability among Kenyan undergraduate students. Journal of Psychology in Africa, 25(3), 216-223. 
Mitchell, K. R., Hayes, M., Gordon, J., \& Wallis, B. (1984). An investigation of the attitudes of medical students to physically disabled people. Medical Education, 18(1), 21-23.

Moroz, A., Gonzalez-Ramos, G., Festinger, T., Langer, K., Zefferino, S., \& Kalet, A. (2010). Immediate and follow-up effects of a brief disability curriculum on disability knowledge and attitudes of PM\&R residents: A comparison group trial. Medical Teacher, 32(8), 360-364.

Mulligan, K., Calder, A., \& Mulligan, H. (2017). Inclusive design in architectural practice: Experiential learning of disability in architectural education. Disability and Health Journal, 11(2), 237-242.

Novo Corti, I., Calvo Babío, N., \& Muñoz Cantero, J. M. (2015). Una perspectiva de género Introducción. Anales de Psicología, 31, 155-171.

Novo Corti, I., \& Muñoz Cantero, J. M. (2015). Los estudiantes universitarios ante la inclusión de sus compañeros con discapacidad: indicadores basados en la teoría de la acción razonada para los estudios de economía y empresa en la Universidad de A Coruña = University students facing inclusión. REOP - Revista Española de Orientación y Psicopedagogía, 23(2), 105.

Oermann, M. H., \& Lindgren, C. L. (1995). An educational program's effects on student's attitudes toward people with disabilities: A 1-Year Follow-Up. Rehabilitation Nursing, 20(1), 6-10.

Oswald, M., \& Swart, E. (2011). Addressing South African pre-service teachers' sentiments, attitudes and concerns regarding Inclusive Education. International Journal of Disability, Development and Education, 58(4), 389-403.

Parasuram, K. (2006). Variables that affect teachers' attitudes towards disability and inclusive education in Mumbai, India. Disability and Society, 21(3), 231-242.

Parkinson, G. (2006). "Counsellors” attitudes towards Disability Equality Training (DET). British Journal of Guidance and Counselling, 34(1), 93-105.

Pernice, R., \& Lys, K. (1996). Interventions for attitude change towards people with disabilities: how successful are they? International Journal of Rehabilitation Research, 19, 171-174.

Richard, I., Compain, V., Mouillie, J. M., Adès, F., Garnier, F., Dubas, F., \& Saint-André, J. P. (2005). Évaluation de l'attitude vis-à-vis des personnes handicapées des étudiants en médecine de $3 \mathrm{e}$ et $4 \mathrm{e}$ année par le questionnaire «attitude towards disabled persons». Effets de l'enseignement théorique et de stages dans les services de médecine physique et ré. Annales de Readaptation et de Medecine Physique, 48(9), 662-667.

Romero-Contreras, S., Garcia-Cedillo, I., Forlin, C., \& Lomelí-Hernández, K. A. (2013). Preparing teachers for inclusion in Mexico: how effective is this process? Journal of Education for Teaching, 39(5), 509-522.

Ryan, J. (2011). Access and participation in higher education of students with disabilities: Access to what? Australian Educational Researcher, 38(1), 73-93.

Sánchez, M. T. P., \& Puerta, M. A. (2018). Primeros pasos hacia la inclusión: actitudes hacia la discapacidad de docentes en educación infantil. Revista de Investigación Educativa, 36(2), 365-379.

Schitko, D., \& Simpson, K. (2012). Hospitality staff attitudes to guests with impaired mobility: the potential of education as an agent of attitudinal change. Asia Pacific Journal of Tourism Research, 17(3), 326-337.

Seccombe, J. A. (2007a). Attitudes towards disability in an undergraduate nursing curriculum: A literature review. Nurse Education Today, 27(5), 459-465. 
Seccombe, J. A. (2007b). Attitudes towards disability in an undergraduate nursing curriculum: The effects of a curriculum change. Nurse Education Today, 27(5), 445-451.

Shields, N., Bruder, A., Taylor, N., \& Angelo, T. (2011). Influencing physiotherapy student attitudes toward exercise for adolescents with Down syndrome. Disability and Rehabilitation, 33(4), 360366.

Smith, N. J., \& McCulloch, J. W. (1978). Measuring attitudes towards the physically disabled: Testing the "Attitude Towards Disabled Persons" scale (A.T.D.P. Form O) on social work and non-social work students. International Journal of Rehabilitation Research, 1(2), 187-197.

Ståhl, C. (2016). Placing people in the same room is not enough: An interprofessional education intervention to improve collaborative knowledge of people with disabilities. Journal of Interprofessional Care, 30(3), 331-337.

Stevens, L. F., Getachew, M. A., Perrin, P. B., Rivera, D., Plaza, S. L. O., \& Arango-Lasprilla, J. C. (2013). Factor analysis of the spanish multidimensional attitudes scale toward persons with disabilities. Rehabilitation Psychology, 58(4), 396-404.

Symons, A. B., McGuigan, D., \& Akl, E. A. (2009). A curriculum to teach medical students to care for people with disabilities: Development and initial implementation. BMC Medical Education, 9(1), $1-7$.

Ten Klooster, P. M., Dannenberg, J. W., Taal, E., Burger, G., \& Rasker, J. J. (2009). Attitudes towards people with physical or intellectual disabilities: Nursing students and non-nursing peers. Journal of Advanced Nursing, 65(12), 2562-2573.

Tindall, D. (2013). Creating disability awareness through sport: exploring the participation, attitudes and perceptions of post-primary female students in Ireland. Irish Educational Studies, 32(4), 457475 .

Tracy, J., \& Graves, P. (1996). Medical students and people with disabilities: A teaching unit for medical students exploring the impact of disability on the individual and the family. Medical Teacher, 18(2), 119-124.

Tracy, J., \& McDonald, R. (2015). Health and disability: Partnerships in action. Journal of Applied Research in Intellectual Disabilities, 28, 22-32.

Tur-Kaspa, H., Weisel, A., \& Most, T. (2000). A multidimensional study of special education students' attitudes towards people with disabilities: A focus on deafness. European Journal of Special Needs Education, 15(1), 13-23.

Uysal, A., Albayrak, B., Koçulu, B., Kan, F., \& Aydin, T. (2014). Attitudes of nursing students toward people with disabilities. Nurse Education Today, 34(5), 878-884.

Van Boxtel, A. M., Napholz, L., \& Gnewikow, D. (1995). Using a wheelchair activity as a learning experience for student nurses. Rehabilitation Nursing, 20(5), 265-272.

VanPuymbrouck, L., \& Friedman, C. (2019). Relationships between occupational therapy students' understandings of disability and disability attitudes. Scandinavian Journal of Occupational Therapy, $27(2), 1-11$.

Velonaki, V. S., Kampouroglou, G., Velonaki, M., Dimakopoulou, K., Sourtzi, P., \& Kalokerinou, A. (2015). Nurses' knowledge, attitudes and behavior toward deaf patients. Disability and Health Journal, 8(1), 109-117. 
Vignes, C., Godeau, E., Sentenac, M., Coley, N., Navarro, F., Grandjean, H., \& Arnaud, C. (2009). Determinants of students' attitudes towards peers with disabilities. Developmental Medicine and Child Neurology, 51(6), 473-479.

Wagner, A. K., \& Stewart, P. J. B. (2001). An internship for college students in Physical Medicine and Rehabilitation - effects on awareness, career choice, and disability perceptions. American Journal of Physical Medicine \& Rehabilitation, 80, 459-465.

Westbrook, M. T., \& Adamson, B. J. (1989). Knowledge and attitudes: aspects of occupational therapy students' perceptions of the handicapped. Australian Occupational Therapy Journal, 36(3), 120-130.

Whiteley, A. D., Kurtz, D. L. M., \& Cash, P. A. (2016). Stigma and developmental disabilities in nursing practice and education. Issues in Mental Health Nursing, 37(1), 26-33.

Willis, D. S., \& Thurston, M. (2015). Working with the disabled patient: Exploring student nurses views for curriculum development using a swot analysis. Nurse Education Today, 35(2), 383-387.

Wilson, J. S., \& Merrill, A. S. (2002). Teaching students to care for and about people with disabilities. Nurse Educator, 27(2), 89-93.

Wong, D. K. P. (2008). Do contacts make a difference? The effects of mainstreaming on student attitudes toward people with disabilities. Research in Developmental Disabilities, 29(1), 70-82.

Wong, D. W., Chan, F., Cardoso, E. da S., \& Miller, S. M. (2004). Attitudes toward people with disabilities in three social contexts: A conjoint analysis. Rehabilitation Counseling Bulletin, 47(4), 194-204.

Woodard, L. J., Havercamp, S. M., Zwygart, K. K., \& Perkins, E. A. (2012). An innovative clerkship module focused on patients with disabilities. Academic Medicine, 87(4), 537-542.

Yorke, A. M., Ruediger, T., \& Voltenburg, N. (2016). Doctor of physical therapy students' attitudes towards people with disabilities: a descriptive study. Disability and Rehabilitation, 39(1), 91-97.

Zychlinski, E., Ben-Ezra, M., \& Raz, Y. H. (2015). Changing attitudes about disability: The impact of the 'Accessible Community' program. Journal of Social Work, 16(6), 742-757.

Recebido em: 09/04/2020

Reformulado em: 14/07/2020

Aprovado em: 24/07/2020 